\title{
Restorative Treatment Patterns After Root Canal Obturation in Public Hospitals in Riyadh
}

\author{
Tariq AlSwayyed ${ }^{1,2}$ \\ Raneem Bin Nafesah ${ }^{2}$ \\ Manal Alqutub ${ }^{3}$ \\ Ali Alfarhan ${ }^{4}$ \\ Arwa Almashhaf ${ }^{5}$ \\ Hessa Alshaikh ${ }^{6}$ \\ Nura Alhedeithi ${ }^{7}$ \\ 'College of dentistry, King Saud bin \\ Abdulaziz University for Health Sciences, \\ Riyadh, Saudi Arabia; ${ }^{2}$ Department of \\ Dentistry, King Abdulaziz Medical City, \\ Riyadh, Saudi Arabia; ${ }^{3}$ Princess Nourah \\ Bint Abdulrahman University, Riyadh, \\ Saudi Arabia; ${ }^{4}$ King Khalid University, \\ Abha, Saudi Arabia; ${ }^{5}$ Ministry of Health, \\ Riyadh, Saudi Arabia; ${ }^{6}$ King Saud bin \\ Abdulaziz University for Health Sciences, \\ Riyadh, Saudi Arabia; ${ }^{7}$ King Saud \\ University, Riyadh, Saudi Arabia
}

Correspondence: Raneem Bin Nafesah King Abdulaziz Medical City, Riyadh, Saudi Arabia

Email ralnafisa@gmail.com
Aim: This study aims to describe the pattern of coronal restoration use among different dental specialties after root canal obturation in two governmental hospitals in Riyadh.

Materials and Methods: Electronic dental records at King Abdul-Aziz Medical City and University Dental Hospital were reviewed. Teeth that underwent initial root canal treatment on a permanent tooth for obturation using gutta-percha between April 2019 and June 2019 were included. The collected data included the type of material used for coronal restoration after RCT, immediate post-space preparation, cotton pellet placement, the clinical title and specialty of the treating physician, and the center where treatment was performed. Excel was used for data collection. IBM SPSS was used for descriptive and interferential analyses.

Results: A total of 763 patients were included in the study, in which the double seal technique was used in $56 \%$ of the patients, followed by Cavit, which was used in approximately $17 \%$ of the patients. Post space was prepared immediately after root canal treatment in 49 patients, and only 17 teeth received the final post, whereas post space was temporized for the rest of the prepared teeth and received the final post at the following visits. Significant relations were found between the type of material used and the clinical title of the treating physician and between the material of choice and the specialty of the dentist. The double seal was the technique of choice among endodontists and restorative dentists, whereas advanced general dentists frequently used Cavit. The least used materials were IRM, amalgam, and Ketac Silver.

Conclusion: The double seal technique was found to be the most commonly used method to achieve a coronal seal, followed by Cavit. Other materials used after RCT, in sequential order based on the frequency of use, were GIC, temporary crowns, resin composite, RMGIC, prefabricated post with composite buildup, IRM, amalgam, and Ketac Silver.

Keywords: coronal restoration, temporary fillings, endodontic treatment, restorative treatment, root canal treatment

\section{Introduction}

Root canal treatment (RCT) aims to promote healing and prevent infection of periapical tissue. ${ }^{1}$ Therefore, to attain the desired outcome of RCTs, microorganisms must be eliminated by thorough cleaning and shaping of the root canal system..$^{2-4}$ Additionally, sealing the root canal system with proper filling material to achieve a "fluid-tight" obturation results in an excellent apical seal, which is essential for the success of the RCT. ${ }^{4,5}$ Endodontic treatment has a high success rate of up to $93 \% .^{6-8}$ However, the lack of adequate coronal coverage significantly reduces treatment success. ${ }^{9}$ Research has found that in the absence of coronal coverage, saliva leaked throughout the whole length of the root filling material in only a week, although the obturation was of good quality. ${ }^{10}$ 
In cases where the canals have been grossly and persistently contaminated, root canal retreatment is required. ${ }^{11}$ Therefore, immediate restorations, whether temporary or permanent, should provide an adequate seal. ${ }^{9,12}$ Temporary restorations are those placed in the access cavity between appointments to provide a coronal seal. ${ }^{13}$ Alongside a good seal, the temporary restoration of choice should provide ease of placement, removal, and adequate strength. ${ }^{12,13}$ Some temporary materials used after endodontic treatment are glass ionomer cement (GIC), resinmodified GIC (RMGIC), reinforced GIC (Ketac Fil and Ketac Silver), composite resins, amalgam, zinc-oxidebased reinforced intermediate restorative material (IRM), calcium sulfate-based filling material (Cavit), and temporary crowns with or without posts.

This study aimed to describe the different types of coronal restorative materials that are directly placed after root canal obturation and compare the usage of several restorative techniques among different dental specialties in King Abdul-Aziz Medical City (KAMC) and University Dental Hospital at King Saud University in Riyadh.

\section{Methods}

Ethical approval was obtained from the institutional review board committee at King Abdullah International Medical Research Center (KAIMRC) in July 2019. Due to the retrospective nature of the study, patient consent was waivered by the IRB. However, patient confidentiality was maintained by keeping the data anonymized in compliance with the Declaration of Helsinki. Documented cases between April 2019 and June 2019 were reviewed. The data were retrieved from electronic dental records in KAMC and University Dental Hospital endodontic, restorative, and advanced general dentistry (AGD) departments. All cases that received complete RCT on a permanent tooth using gutta-percha as obturating material were included in the study. No gender restriction was applied. Records of patients were excluded if they were younger than 15 years of age, had incomplete documentation, underwent retreatment, or underwent a treatment regiment in which materials other than gutta-percha were used. Excel was used by trained research members to collect the data. The collected data included the type of material used for coronal restoration after RCT, whether or not post space was immediately prepared after obturation, cotton pellet placement before temporization, the clinical title and specialty of the treating physician, and the center where treatment was performed. The raw dataset was compiled, validated, and checked for missing values or outliers. Then, cases were deidentified and anonymized to maintain confidentiality. The data analysis process of this study included two stages. The first stage included descriptive analysis, where all categorical variables were described as frequencies and percentages. The second stage included hypothesis testing using the Pearson chi-square test. The test was applied using IBM SPSS Statistics 25.0. A relationship was considered significant if the $\mathrm{P}$ value was $5 \%$ ( $\alpha$ value) or less along with a confidence interval of $95 \%$ and a $\beta$ value of 0.2 .

\section{Results}

A total of 1020 electronic dental records of patients who underwent RCT were reviewed. Only 763 records met the inclusion criteria and were included in the study. Table 1 demonstrates the clinical title and specialty of the treating dentist as well as the hospital of treatment. Most patients were treated at the University Dental Hospital, which accounted for more than half of the patients. The records also showed that most of the treatment was performed by endodontists $(85.1 \%)$, followed by restorative dentists and advanced general dentists (AGDs). Moreover, residents and consultants were found to have performed most of the treatment, whereas specialists performed only approximately $8 \%$ of the treatment.

Table 2 shows the pattern of usage of different restorative materials for coronal seal, post-space preparation, and cotton pellet placement. This demonstrates that Ketac Silver, amalgam, and IRM were among the least used materials after RCT. These materials were collectively used in less than $0.8 \%$ of the patients. On the other hand, the double seal technique was used in more than half of the patients. Moreover, a cotton pellet was placed in approximately $40 \%$ of the patients, and post space was prepared in only approximately $6 \%$ of the treated teeth.

Table 3 demonstrates the distribution of using restorative materials among different specialties, and it shows the significant relations found between the variables. The double seal was the most commonly used technique among endodontists and restorative dentists, whereas Cavit and GIC were the most commonly used materials by AGDs. Moreover, significant relations were found between the type of material used and the dentist's specialty, between the material used and the dentist's clinical title, and between the material used and hospital where the treatment was performed, with $\mathrm{P}$ values of $0.000,0.001$, and 0.000 , respectively. 
Table I Hospital of Treatment, Clinical Title and Specialty of the Treating Dentist

\begin{tabular}{|l|c|}
\hline Variable & $\mathbf{n}(\%)$ \\
\hline Hospital & \\
KSU & $504(66.1)$ \\
NGHA & $259(33.9)$ \\
\hline Dentist's specialty & \\
AGD & $39(5.1)$ \\
Restorative dentist & $75(9.8)$ \\
Endodontist & $649(85.1)$ \\
\hline Dentist's clinical title & \\
Resident & $353(46.3)$ \\
Specialist & $60(7.9)$ \\
Consultant & $350(45.9)$ \\
\hline
\end{tabular}

Table 2 Pattern of Restorative Materials Usage for Coronal Seal, Cotton Pellet Placement, and Post Space Preparation

\begin{tabular}{|l|c|}
\hline Variable & n (\%) \\
\hline Material type & $37(4.8)$ \\
RMGIC & $129(16.9)$ \\
Cavit & $3(0.4)$ \\
IRM & $1(0.1)$ \\
Ketac Silver & $58(7.6)$ \\
GIC & $39(5.1)$ \\
Composite & $427(56.0)$ \\
Double seal & $17(2.2)$ \\
Post with composite buildup & $50(6.6)$ \\
Temporary crown & $2(0.3)$ \\
Amalgam & \\
\hline Cotton pellet placement & $295(38.7)$ \\
Placed & $468(61.3)$ \\
Not placed & \\
\hline Post space preparation & $49(6.4)$ \\
Prepared & $714(93.6)$ \\
Not prepared & \\
\hline
\end{tabular}

\section{Discussion}

Many factors determine the prognosis of endodontically treated teeth. ${ }^{14}$ Factors that are related to each patient could contribute to the success or failure of endodontic treatment. ${ }^{14}$ Nevertheless, the basic biologic principle that has been demonstrated by Kakehashi et al still stands. ${ }^{15}$ Apical periodontitis is caused mainly by the presence of bacteria. ${ }^{15}$ Therefore, thorough cleaning and shaping of the canals and well-sealed fillings are essential to increase the chances of clinical success of the treatment. Multiple studies have argued the importance of a coronal seal over a

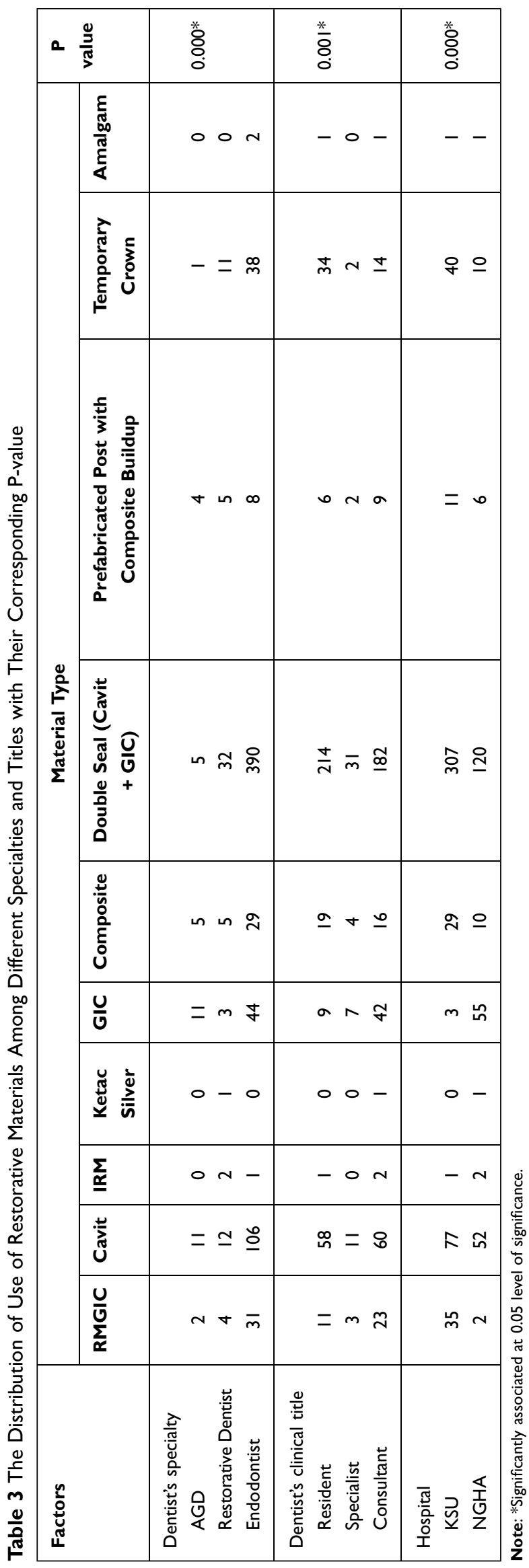


canal seal, ${ }^{16-18}$ while other studies have discussed the opposite association; ${ }^{19-21}$ However, a systematic review conducted by Gillen et al concluded that the quality of both root canal filling, as well as the quality of coronal restoration, is of the utmost importance to increase the chance of success of the treatment. ${ }^{22}$ This retrospective study aimed to describe and compare the usage of the types of coronal restorative materials that are placed directly after root canal obturation among dental specialties in King Abdul-Aziz Medical City and University Dental Hospital at King Saud University in Riyadh. Electronic dental records in both institutions were reviewed, and 763 patient records were included in the study.

In patients where teeth were temporized, cotton pellets were placed in almost $40 \%$ of the teeth. The use of cotton pellets over the canal orifice is controversial. ${ }^{12}$ Their use facilitates the subsequent removal of the temporary restoration, and it prevents the restoration from blocking the canals. ${ }^{12}$ On the other hand, their use can reduce the thickness of the overlaying restoration, which compromises the seal and may affect the restoration's strength. ${ }^{23,24}$ Moreover, cotton pellets may allow displacement of the restoration during mastication by acting as a cushion. Additionally, microscopic cotton fibers may be inadvertently exposed to the oral environment and produce a wicking effect of saliva and bacteria. Finally, the use of cotton pellets may cause leakage through exposed dentinal tubules. ${ }^{23,24}$ Therefore, care should be taken when placing cotton pellets. Using a small well-adapted piece of cotton over the orifice and not the chamber is advised. ${ }^{12}$ Alternatively, a sterile well-adapted part of polytetrafluoroethylene (PTFE) tape could be used. ${ }^{24}$

In this study, post space was prepared immediately after obturation in 49 teeth, and only 17 teeth received the final post, whereas the rest of the teeth were temporized. An ideal seal of the canal can be obtained by immediate placement of the permanent post and core. ${ }^{13}$ In contrast, temporary posts are not effective in preventing leakage. ${ }^{25,26}$ In fact, it has been shown that the use of a temporary post allows as much contamination as when no post is present. ${ }^{26}$

Significant relations were found between the type of material used and the clinical title of the dentist and between the type of material used and the dentist's specialty. Cavit and GIC were the most commonly used materials among AGDs, as they were used in $56.4 \%$ of the patients. Cavit is a zinc oxide/calcium sulfate preparation. ${ }^{12}$ Because of its hygroscopic properties (water absorption), it provides an excellent marginal seal; ${ }^{12}$ however, an in vitro study conducted by Pieper et al detected dye penetration through the bulk of the material, which confirms previous findings by Cruz et al. ${ }^{27,28}$ This suggests that recontamination could occur by bacterial infiltration through the material itself. Therefore, an adequate bulk of the material is required to reduce bacterial microleakage and overcome the low compressive strength of Cavit. $^{12,13}$ A $3.5-4 \mathrm{~mm}$ thickness of the material is recommended. ${ }^{12,13}$ The hygroscopic property of Cavit may also pose a disadvantage, as evidence has shown that this property might lead to cuspal deflection and tooth fracture. ${ }^{29}$

GIC is an adhesive restorative material that bonds to the tooth through a chelation reaction that occurs between the calcium group in the tooth and the carboxyl group in GIC, creating a chemical bond. ${ }^{30}$ An in vitro study conducted to assess the sealability of GIC at different periods concluded that GIC provided a superior seal compared to that of other materials, yet seal deterioration occurred at two weeks and was significant after four weeks. ${ }^{31}$ Alongside its adhesion to the tooth structure, other advantages of GIC include fluoride release and antibacterial activity, which is owed to its low $\mathrm{pH}$ and the presence of certain cations. ${ }^{12}$ Some GIC disadvantages include difficulty in differentiating it from the surrounding tooth structure, cost compared to other materials, and susceptibility to shrinkage during setting, which compromises the coronal seal. $^{12,13,32}$

To overcome the shortcomings of temporary restorative materials, a double seal technique was proposed. ${ }^{33}$ In this technique, two temporary materials are placed on top of each other as an internal layer and an external layer, thus enabling more adaptation to cavity walls and resulting in less leakage. ${ }^{33}$ Commonly used combinations include Cavit as the inner material, IRM as the outer material or Cavit and GIC. ${ }^{12,13,33}$ In this study, the use of Cavit and GIC was referred to as a double seal. It was found that this combination was the most commonly used coronal restorative technique among restorative dentists and endodontists; also, it was the most widely used technique across dentists with all clinical titles, as approximately $56 \%$ of all the patients were restored utilizing this technique.

IRM, Ketac Silver, and amalgam were among the least used materials. IRM is a zinc oxide eugenol-based material reinforced with polymethyl methacrylate. Due to the presence of eugenol, IRM exhibits antibacterial activity. ${ }^{12}$ However, the presence of eugenol impairs the polymerization of resin composites. ${ }^{27}$ Furthermore, research has shown that IRM has a poor marginal seal, performs poorly 
under stress, and exhibits bacterial penetration through the bulk of the material. ${ }^{27,31,33}$ In a study conducted by Udayakumar et al, it was found that after 7 days, maximum leakage was detected in IRM samples compared to that observed with the use of other materials. After 18 days, leakage was detected in $100 \%$ of the IRM-restored samples. ${ }^{34}$ Another study stated that "IRM resulted in the poorest marginal seal" compared to Cavit, GIC, and dimethacrylate-based filling. ${ }^{27}$ These findings may explain its scanty usage.

To our knowledge, this paper is the first to study restorative material usage after root canal obturation in Saudi Arabia; nonetheless, one of the shortcomings of this paper is that data were collected in two governmental hospitals and learning institutions. Materials used in private practice, where the cost of the material is a major determining factor for its use, may follow a different distribution. Other studies in various areas of Saudi Arabia and among different types of practices are required for additional data comparisons and for the construction of universal guidelines.

\section{Conclusion}

Many factors can affect the success of endodontic treatment. One of the main factors is the coronal seal; thus, the coronal restoration of choice should have adequate strength and an excellent seal. In this study, it was found that the double seal technique, which combines the advantages of two types of temporary restorations to overcome the disadvantages of each individual type, was the most commonly used method to achieve a coronal seal, followed by the use of Cavit. Cavit was the second most widely used material for restorative dentists and endodontists and the first for AGDs together with GIC. Other materials that were found to be used after RCT, listed following their frequency of use, were temporary crowns, composite resin, RMGIC, prefabricated post with composite buildup, IRM, amalgam, and Ketac Silver. Ketac Silver was the least used material, as it was used only once throughout the sample.

Moreover, post space was prepared in 49 teeth and was temporized in 32 of them; post space temporization was reported in the literature as ineffective in preventing leakage. ${ }^{25,26}$ Cotton pellets were placed in less than $40 \%$ of teeth before temporization. Placement cotton pellet before temporization is controversial. ${ }^{12,23,24}$
This study provides baseline information that could aid in establishing guidelines for selecting the best material to provide patients with the best possible treatment outcomes.

\section{Disclosure}

The authors report no conflicts of interest in this work.

\section{References}

1. Ørstavik D. Essential Endodontology: Prevention and Treatment of Apical Periodontitis. 3rd ed. John Wiley \& Sons Ltd; 2019:59-90.

2. European Society of Endodontology. Quality guidelines for endodontic treatment: consensus report of the European Society of Endodontology. Int Endod J. 2006;39(12):921-930. doi:10.1111/ j.1365-2591.2006.01180.x

3. Lin P, Huang S, Chang H, Chi L. The effect of rubber dam usage on the survival rate of teeth receiving initial root canal treatment: a Nationwide Population-based Study. J Endod. 2014;40(11):17331737. doi:10.1016/j.joen.2014.07.007

4. Mandke L. Importance of coronal seal: preventing coronal leakage in endodontics. $J$ Restor Dent. 2016;4(3):71. doi:10.4103/23214619.188224

5. Muliyar S, Shameem KA, Thankachan RP, Francis PG, Jayapalan CS, Hafiz KA. Microleakage in endodontics. J Int Oral Health. 2014;6(6):99-104.

6. Elemam R, Pretty I. Comparison of the success rate of endodontic treatment and implant treatment. ISRN Dent. 2011;2011:1-8. doi:10.5402/2011/640509

7. De Chevigny C, Dao T, Basrani B, et al. Treatment outcome in endodontics: the Toronto Study_phases 3 and 4: orthograde retreatment. J Endod. 2008;34(2):131-137. doi:10.1016/j.joen.2007.11.003

8. De Chevigny C, Dao $\mathrm{T}$, Basrani B, et al. Treatment outcome in endodontics: the Toronto Study-phase 4: initial treatment. $J$ Endod. 2008;34(3):258-263. doi:10.1016/j.joen.2007.10.017

9. Cheung G, Chan T. Long-term survival of primary root canal treatment carried out in a dental teaching hospital. Int Endod J. 2003;36 (2):117-128. doi:10.1046/j.1365-2591.2003.00639.x

10. Balto H. An assessment of microbial coronal leakage of temporary filling materials in endodontically treated teeth. J Endod. 2002;28 (11):762-764. doi:10.1097/00004770-200211000-00004

11. Vârlan C, Dimitriu B, Vârlan V, Bodnar D, Suciu I. Current opinions concerning the restoration of endodontically treated teeth: basic principles. J Med Life. 2009;2:165-172.

12. Naoum H, Chandler N. Temporization for endodontics. Int Endod J. 2002;35(12):964-978. doi:10.1046/j.1365-2591.2002.00600.x

13. Eliyas S, Jalili J, Martin N. Restoration of the root canal treated tooth. Br Dent J. 2015;218(2):53-62. doi:10.1038/sj.bdj.2015.27

14. Song M, Jung I, Lee S, Lee C, Kim E. Prognostic factors for clinical outcomes in endodontic microsurgery: a Retrospective Study. $J$ Endod. 2011;37(7):927-933. doi:10.1016/j.joen.2011.04.005

15. Kakehashi S, Stanley H, Fitzgerald R. The effects of surgical exposures of dental pulps in germ-free and conventional laboratory rats. Oral Surg Oral Med Oral Pathol. 1965;20(3):340-349. doi:10.1016/ 0030-4220(65)90166-0

16. Ray H, Trope M. Periapical status of endodontically treated teeth in relation to the technical quality of the root filling and the coronal restoration. Int Endod J. 1995;28(1):12-18. doi:10.1111/j.13652591.1995.tb00150.x

17. Hommez G, Coppens C, De Moor R. Periapical health related to the quality of coronal restorations and root fillings. Int Endod J. 2002;35 (8):680-689. doi:10.1046/j.1365-2591.2002.00546.x 
18. Kayahan M, Malkondu Ö, Canpolat C, Kaptan F, Bayırlı G, Kazazoglu E. Periapical health related to the type of coronal restorations and quality of root canal fillings in a Turkish subpopulation. Oral Surg Oral Med Oral Pathol Oral Radiol Endod. 2008;105(1): e58-e62. doi:10.1016/j.tripleo.2007.07.044

19. Tavares P, Bonte E, Boukpessi T, Siqueira J, Lasfargues J. Prevalence of apical periodontitis in root canal-treated teeth from an urban French population: influence of the quality of root canal fillings and coronal restorations. J Endod. 2009;35(6):810-813. doi:10.1016/j. joen.2009.03.048

20. Song M, Park M, Lee C, Kim E. Periapical status related to the quality of coronal restorations and root fillings in a Korean population. J Endod. 2014;40(2):182-186. doi:10.1016/j.joen.2013.10.017

21. Siqueira J, Rôças I, Alves F, Campos L. Periradicular status related to the quality of coronal restorations and root canal fillings in a Brazilian population. Oral Surg Oral Med Oral Pathol Oral Radiol Endod. 2005;100(3):369-374. doi:10.1016/j.tripleo.2005.03.029

22. Gillen B, Looney S, Gu L, et al. Impact of the quality of coronal restoration versus the quality of root canal fillings on success of root canal treatment: a systematic review and meta-analysis. $J$ Endod. 2011;37(7):895-902. doi:10.1016/j.joen.2011.04.002

23. Bishop K, Briggs P. Endodontic failure-a problem from top to bottom. Br Dent J. 1995;179(1):35-36. doi:10.1038/sj.bdj.4808813

24. Sattar M, Patel M, Alani A. Clinical applications of polytetrafluoroethylene (PTFE) tape in restorative dentistry. Br Dent J. 2017;222 (3):151-158. doi:10.1038/sj.bdj.2017.110

25. Schwartz R, Robbins J. Post placement and restoration of endodontically treated teeth: a literature review. J Endod. 2004;30(5):289-301. doi:10.1097/00004770-200405000-00001

26. Demarchi M, Sato E. Leakage of interim post and cores used during laboratory fabrication of custom posts. J Endod. 2002;28(4):328-329. doi:10.1097/00004770-200204000-00017
27. Pieper C, Zanchi C, Rodrigues-Junior S, Moraes R, Pontes L, Bueno M. Sealing ability, water sorption, solubility and toothbrushing abrasion resistance of temporary filling materials. Int Endod J. 2009;42 (10):893-899. doi:10.1111/j.1365-2591.2009.01590.x

28. Cruz E, Shigetani Y, Ishikawa K, Kota K, Iwaku M, Goodis H. A laboratory study of coronal microleakage using four temporary restorative materials. Int Endod J. 2002;35(4):315-320. doi:10.1046/j.1365-2591.2002.00446.x

29. Laustsen M, Munksgaard E, Reit C, Bjorndal L. A temporary filling material may cause cusp deflection, infractions and fractures in endodontically treated teeth. Int Endod J. 2005;38(9):653-657. doi:10.1111/j.1365-2591.2005.01003.x

30. Culbertson B. Glass-ionomer dental restoratives. Prog Polym Sci. 2001;26(4):577-604. doi:10.1016/S0079-6700(01)00006-5

31. Madarati A, Rekab M, Watts D, Qualtrough A. Time-dependence of coronal seal of temporary materials used in endodontics. Aust Endod J. 2008;34(3):89-93. doi:10.1111/j.1747-4477.2007.00079.x

32. Divya KT, Satish G, Srinivasa TS, Reddy V, Umashankar K, Rao BM. Comparative evaluation of sealing ability of four different restorative materials used as coronal sealants: an in vitro study. $J$ Int Oral Health. 2014;6(4):12-17.

33. Pai S, Yang S, Sue W, Chueh L, Rivera E. Microleakage between endodontic temporary restorative materials placed at different times. J Endod. 1999;25(6):453-456. doi:10.1016/S0099-2399(99)80278-7

34. Udayakumar P, Kaushik M, Prashar N, Arya S. Coronal leakage of provisional restorative materials used in endodontics with and without intracanal medication after exposure to human saliva. Saudi Endod J. 2016;6(2):77. doi:10.4103/1658-5984.180620

\section{Publish your work in this journal}

Clinical, Cosmetic and Investigational Dentistry is an international, peer-reviewed, open access, online journal focusing on the latest clinical and experimental research in dentistry with specific emphasis on cosmetic interventions. Innovative developments in dental materials, techniques and devices that improve outcomes and patient satisfaction and preference will be highlighted. The manuscript management system is completely online and includes a very quick and fair peer-review system, which is all easy to use. Visit http://www.dovepress.com/testimonials.php to read real quotes from published authors. 